\section{THE INTERNATIONAL REGISTRY FOR NIEMANN-PICK DISEASE TYPE C (NP-C) IN CLINICAL PRACTICE}

doi:10.1136/archdischild-2012-302724.1035

${ }^{1} F A$ Wijburg, ${ }^{2} E$ Mengel, ${ }^{3} \mathrm{MC}$ Patterson, ${ }^{4} \mathrm{JE}$ Wraith, ${ }^{5} \mathrm{MT}$ Vanier, ${ }^{6} \mathrm{~B}$ Schwierin, ${ }^{6} \mathrm{~A}$ Muller, ${ }^{7} \mathrm{H}$ Drevon, ${ }^{8} \mathrm{M}$ Pineda. ${ }^{1}$ Department of Pediatrics, Academic Medical Center, University of Amsterdam, Amsterdam, The Netherlands, ' ${ }^{2}$ Villa Metabolica, University of Mainz, Mainz, Germany; ${ }^{3}$ Mayo Clinic, Rochester, MN, USA; ${ }^{4}$ Academic Health Science Centre, St Mary's Hospital, Manchester, UK; ${ }^{5}$ NSSERM Unit 820, Lyon, France; ${ }^{6}$ Actelion Pharmaceuticals Ltd, Allschwil, Switzerland; 'Numerus Ltd, Sandhurst, UK; ${ }^{8} \mathrm{Hospital}$ Sant Joan de Déu, Barcelona, Spain

Background and Aim An international disease registry was started in September 2009 to evaluate the long-term disease course of NP-C in clinical settings.

Methods Descriptive data from enrolment are presented for all patients with available data who were included in the Registry as of $19^{\text {th }}$ August 2011

Results 121 patients have been enrolled. The median (range) age at enrolment was $16.9(0.9-56.6)$ years, age at onset of neurological manifestations was $8.2(<1-48.0)$ years $(n=100)$, and age at diagnosis was $11.8(0.1-53.9)$ years $(n=110)$. A history of neonatal jaundice was recorded in $4 / 4$ evaluable patients with early-infantile (EI) onset of neurological manifestations (at age < 2 years; $n=9$ ), $6 / 21$ $(29 \%)$ with late-infantile (LI) onset (at 2 to $<6$ years; $n=31$ ), $6 / 21$ $(29 \%)$ with juvenile (JUV) onset (at 6 to $<15$ years; $n=31$ ), and $3 / 20$ $(15 \%)$ with adolescent/adult (AA) onset (at $\geq 15$ years; $n=29$ ). Miglustat therapy at enrolment was recorded in 88/121 (73\%) patients; mean (SD) exposure 1.69 (1.85) years ( $\mathrm{n}=86)$. Neurological manifestations were observed in $71 / 84(85 \%)$ patients: ataxia (71\%), vertical gaze palsy (68\%) and dysarthria (62\%) were most frequent. Median (range) disability scores $(0=$ normal; $1=$ worst) were: $0.0(0.0-0.94)$ in EI ( $n=7), 0.29(0.0-1.0)$ in LI ( $n=28), 0.41$ $(0.15-0.88)$ in JUV $(n=28)$, and $0.29(0.06-0.81)$ in AA-onset patients $(n=26)$. A low proportion of patients had normal language, manipulation, ambulation, and/or swallowing.

Conclusions Over two-thirds of this NP-C cohort had infantile or juvenile onset of neurological manifestations; neonatal jaundice was observed more frequently in these patients versus adolescent/ adult-onset patients.

\section{GRACILE SYNDROME IN A TURKISH NEWBORN INFANT CAUSED BY A HOMOZYGOUS MUTATION (P99L) IN COMPLEX III ASSEMBLY FACTOR BCS1L}

doi:10.1136/archdischild-2012-302724.1036

'E Serdaroglu, ${ }^{2} \mathrm{~S}$ Takci, ${ }^{3} \mathrm{H}$ Kotarsky, ${ }^{1} \mathrm{O}$ Cil, ${ }^{4} \mathrm{E}$ Utine, ${ }^{2} \mathrm{~S}$ Yigit, $5,6 \mathrm{~V}$ Fellman. ${ }^{1}$ Department of Pediatrics; ${ }^{2}$ Department of Pediatrics, Division of Neonatology, Hacettepe University, Ankara, Turkey; ${ }^{3}$ Department of Pediatrics, Clinical Sciences, Lund University, Lund, Sweden; ${ }^{4}$ Department of Pediatrics, Division of Medical Genetics, Hacettepe University, Ankara, Turkey; ${ }^{5}$ Department of Pediatrics, Lund University and Skåne University Hospital Lund, Lund, Sweden; ${ }^{6}$ Department of Pediatrics, University of Helsinki, Helsinki University Central Hospital, Helsinki, Finland

Background and Aim GRACILE syndrome, a neonatal, autosomally recessive disorder found in Finland, featuring growth retardation, aminoaciduria, cholestasis, iron overload, lactic acidosis and early death, is caused by a homozygous mutation (S78G) in BCS1L, the assembly factor for respiratory chain complex III. We investigated a newborn Turkish girl with similar symptoms. Her two sisters with low birth weight, metabolic acidosis, cholestasis and renal Fanconi syndrome, had died at 18 and 105 days age, respectively.

Methods and results The girl was born to healthy nonconsanguineous parents. She was growth retarded (1789 g at term), developed tachypnea and metabolic acidosis on day one. Lactic acidosis, jaundice with direct hyperbilirubinemia, nonspecific aminoaciduria, high phosphaturia, proteinuria and glucosuria were detected. Serum iron $(190 \mathrm{mcg} / \mathrm{dl})$, ferritin $(2819 \mathrm{ng} / \mathrm{ml})$ and transferrin saturation (99.4\%) were increased. Metabolic, cardiologic and sonographic workup were otherwise normal. Because of similarities with GRACILE syndrome, the BCS1L gene was investigated. The Finnish SNP was not found, but gene sequencing revealed a homozygous mutation resulting in an amino acid exchange (P99L) in the protein.

Conclusions The studied infant had a GRACILE-like disorder caused by a different mutation than that in newborns of Finnish ancestors. Most likely the two diseased siblings had the same homozygous BCS1L mutation that previously has been published in three other newborns or Turkish origin. We proposed that P99L-mutation in BCS1L is a Turkish genotype resulting in GRACILE syndrome phenotype, and should be investigated in Turkish newborns with the typical clinical features.

\section{DETERMINATION OF PREALBUMIN, SELENIUM, ZINC AND IRON CONCENTRATION IN SERUM FOR MONITORING THE NUTRITION STATUS OF PHENYLKETONURIC AND HYPERPHENYLALANINEMIC PATIENTS}

doi:10.1136/archdischild-2012-302724.1037

'D Prochazkova, 'L Kolbova, ${ }^{2} \mathrm{~J}$ Jarkovsky, ${ }^{3} \mathrm{H}$ Vinohradska, ${ }^{1 P}$ Konecna, ${ }^{1} \mathrm{Z}$ Dolezel. ${ }^{1} \mathrm{Dpt}$. of Pediatrics; 'Institute of Biostatistic and Analyses; ${ }^{3}$ Dpt. of Biochemistry, Medical Faculty of Masaryk University and University Hospital Brno, Brno, Czech Republic

Background and Aims Phenylketonuria is an inherited disorder of metabolism of the amino acid phenylalanine caused by a deficit of the enzyme phenylalaninhydroxylase. It is treated with a lowprotein diet containing a low content of phenylalanine to prevent mental affection of the patient. The objective of the present study was to assess the compliance of our phenylketonuric (PKU) and hyperphenylalaninemic (HPA) patients; to determine the concentration of serum pre-albumin and trace elements to discover the potential correlation between the amount of proteins in food and their metabolic control.

Methods The prospective study contained altogether 174 patients, of which 113 were children, 60 with PKU and 53 with HPA and 61 were adults, 51 with PKU and 10 with HPA.

Results We did not prove a statistically significant difference in the levels of serum pre-albumin, zinc and iron among the respective groups. We proved statistically significant difference in the level of serum selenium among PKU and HPA patients in adulthood ( $\mathrm{p}=0.006$, Mann-Whitney $\mathrm{U}$ test).

Conclusion The therapeutic restrictive diet for PKU and HPA makes the patient liable to the risk of nutritional deficit.

\section{UREA CYCLE DEFECTS- MISDIAGNOSES AND WRONG DIAGNOSES}

doi:10.1136/archdischild-2012-302724.1038

S Abed. Pediatrics, Naser Pediatric Hospital, Gaza, Palestinian Authority

Background Urea cycle defects (UCD) constitute a group of rare metabolic disorders that involve the enzymes of every step of urea cycle. Deficiency of one of these enzymes leads to hyperammonemia and they present classically with acute life-threatening neonatal encephalopathy. However, presentation at later childhood or adulthood could also occur. There are many disorders that mimic UCD causing misdiagnosis or wrong diagnosis.

Methods A prospective and retrospective study was made on 10 cases of UCD. Most have been diagnosed at the neonatal period with follow up done through our genetic and metabolic clinic at Naser Pediatric Hospital.

Results Most of the cases presented with acute ammonia encephalopathy. Age of presentation was variable. Most of the cases were from the Northern Gaza which is of geographical similarity to distribution of the IEM collectively. There was no gender differences. 